\title{
Efectividad, seguridad y tolerancia del balón intragástrico asociado a una dieta hipocalórica para la reducción de peso en pacientes obesos
}

\author{
A. Escudero Sanchis, I. Catalán Serra, J. Gonzalvo Sorribes, M. Bixquert Jiménez, L. Navarro López, \\ L. Herrera García, L. Durbán Serrano y A. Monforte Albalat
}

Servicio de Digestivo. Hospital Arnau de Vilanova. Valencia

\section{RESUMEN}

Introducción: la implantación de un balón intragástrico (BI) por vía endoscópica asociado a una dieta hipocalórica es una alternativa en el tratamiento de la obesidad. El objetivo de nuestro estudio es evaluar su efectividad, seguridad y tolerancia.

Material y métodos: se incluyen de manera prospectiva 38 pacientes sin contraindicaciones para la implantación del BI, desde marzo de 2004 hasta enero de 2007. La retirada del balón se realizó 6 meses después de la implantación. Evaluamos el peso e índice de masa corporal (IMC) tras la retirada del balón, a los 6 y 12 meses postretirada, así como la tolerancia y aparición de complicaciones durante el tratamiento. Tras la retirada del balón se realizó un cuestionario a cada paciente evaluando la percepción subjetiva al tratamiento.

Resultados: después de 6 meses de tratamiento la pérdida de peso media fue de $14,10 \mathrm{~kg}(0-46)$ y la reducción media del IMC fue de $5,23 \mathrm{~kg} / \mathrm{m}^{2}(0-18)$. A los 12 meses postretirada del balón el $48,4 \%$ de pacientes mantiene o sigue perdiendo peso. Los síntomas precoces más frecuentes fueron náuseas $(71,1 \%)$ y vómitos (57,9\%), con buena respuesta a tratamiento sintomático. Presentaron complicaciones 7 pacientes $(18,4 \%)$ : intolerancia digestiva en 4 pacientes, requiriendo retirada precoz del balón en 3 de ellos; esofagitis moderada en 2 pacientes; y perforación gástrica complicada con shock séptico y exitus en 1 paciente.

\section{Conclusiones:}

1. El balón intragástrico asociado a una dieta hipocalórica puede considerarse un tratamiento efectivo, seguro y bien tolerado para el tratamiento de pacientes con obesidad mórbida.

2. La pérdida de peso se mantiene en casi la mitad de los pacientes al año tras la retirada del balón.

Palabras clave: Obesidad. Obesidad mórbida. Balón intragástrico. Endoscopia.

\begin{abstract}
Introduction: the endoscopic placement of an intragastric balloon (IGB) in association with a low-calorie diet is an option for the treatment of obesity. The aim of the present study was to evaluate its effectiveness, safety, and tolerance.

Material and methods: thirty-eight patients with no contraindications for IGB were included in this prospective study from March 2004 to January 2007. Balloon removal was performed 6 months later. Weight and body mass index (BMI) were evaluated after IGB removal and at 6 months and 1 year thereafter. Tolerance and complications during treatment were evaluated. Patients filled out a questionnaire to evaluate their subjective perception of treatment.

Results: mean weight loss after 6 months on balloon treatment was $14.10 \mathrm{~kg}(0-46)$, and mean BMI reduction was 5.23 $\mathrm{kg} / \mathrm{m} 2(0-18)$. At 12 months after balloon removal $48.4 \%$ of patients maintained their weight loss or kept loosing weight. Most common early symptoms included nausea (71.1\%) and vomiting $(57.9 \%)$ with a good response to symptomatic treatment. Complications were seen in 7 patients (18.4\%): digestive intolerance in 4 patients, with early removal in 3 of them; moderate esophagitis in 2 patients; and gastric perforation complicated with septic shock and death in 1 patient.

Conclusions:

1. IGB in association with low-calorie diet is an effective, safe, and well tolerated treatment for morbid obese patients.

2. Almost half of patients maintained their weight loss after one year from balloon removal.
\end{abstract}

Key words: Obesity. Morbid obesity. Intragastric balloon. Endoscopy.

Escudero Sanchis A, Catalán Serra I, Gonzalvo Sorribes J, Bixquert Jiménez M, Navarro López L, Herrera García L, Durbán Serrano $L$, Monforte Albalat A. Efectividad, seguridad y tolerancia del balón intragástrico asociado a una dieta hipocalórica para la reducción de peso en pacientes obesos. Rev Esp Enferm Dig 2008; 100: 349-354. 


\section{INTRODUCCIÓN}

La obesidad constituye uno de los problemas de salud pública más importantes en razón a su prevalencia y a sus potenciales complicaciones asociadas (1).

Actualmente dentro de las opciones terapéuticas de la obesidad el primer escalón consiste en la combinación de una dieta hipocalórica y cambios en el estilo de vida $(2,3)$, así como tratamiento farmacológico de forma temporal. El tratamiento quirúrgico queda reservado al fracaso del tratamiento médico.

El balón intragástrico (BI) es un tratamiento utilizado no sólo para conseguir una pérdida de peso en pacientes obesos, sino también un mecanismo para disminuir los factores de riesgo asociados a la obesidad previamente a la cirugía bariátrica (4).

El BI ha sido propuesto para las siguientes indicaciones $(5,6)$ : a) pacientes obesos refractarios a tratamiento dietético; b) uso temporal prequirúrgico para obtener una pérdida de peso y reducir el riesgo de la intervención quirúrgica $(7,8)$; y c) pacientes con obesidad grave no candidatos a cirugía de obesidad.

La idea de utilizar un BI en el tratamiento de la obesidad, produciendo sensación de saciedad, fue llevada a cabo por primera vez en 1982; el concepto fue desarrollado a partir de las observaciones realizadas en pacientes con bezoares gástricos (9). Sin embargo entre 19871989 esta técnica fue abandonada debida al alto número de complicaciones y ausencia de eficacia (10), haciendo necesaria la definición de las características del BI ideal (11).

Nuestro estudio evalúa el tratamiento de la obesidad con el BI: su efectividad (pérdida de peso tras retirada y a medio plazo), tolerancia y complicaciones, así como satisfacción del paciente tras el tratamiento.

\section{MATERIAL Y MÉTODOS}

Desde marzo de 2004 hasta enero de 2007 fueron incluidos de forma prospectiva 38 pacientes obesos, refractarios a tratamiento hipocalórico habitual, en los que el Servicio de Endocrinología sentó la indicación de colocación del BI. Se obtuvo el consentimiento informado escrito de cada paciente.

Desde 1998 disponemos del balón intragástrico Bioenterics $^{\circledast}$ (BIB) que reúne las características ideales $(12,13)$ : esférico y de superficie lisa, relleno de líquido, cubierta externa de silicona resistente al ácido gástrico y contiene un marcador radioopaco que permite un seguimiento adecuado.

Previamente a la inserción del BIB se realizó a todos los pacientes: una historia clínica completa (historia médica, enfermedades asociadas, hábitos dietéticos, tratamientos previos, historia psicológica y social), una analítica completa (hemograma, coagulación, glucosa, urea, creatinina, sodio, potasio, albúmina, proteínas totales, tri- glicéridos, colesterol, transaminasas, fosfatasas alcalinas y hormonas tiroideas), una endoscopia digestiva alta y valoración por Psiquiatría descartando cualquier alteración que contraindique su colocación.

De los 38 pacientes incluidos, 11 eran hombres $(28,9 \%)$ y 27 mujeres $(71,1 \%)$; la edad media fue de 40,47 (23-64) con un peso medio inicial de $125,32 \mathrm{~kg}$ (86,50-210), siendo el peso medio inicial en mujeres de $117,60 \mathrm{~kg}$ y en hombres $144,25 \mathrm{~kg}$. El IMC medio inicial fue $47,25 \mathrm{~kg} / \mathrm{m}^{2}$ (34-80), siendo el IMC medio inicial en mujeres $47,17 \mathrm{~kg} / \mathrm{m}^{2}$ y en hombres $47,42 \mathrm{~kg} / \mathrm{m}^{2}$ (Tabla I).

Los criterios de inclusión fueron:

- Pacientes obesos (IMC $>30 \mathrm{~kg} / \mathrm{m}^{2}$ ) mayores de edad (> 18 años).

- Refractarios a tratamiento hipocalórico.

- Con consentimiento informado.

- Informe psiquiátrico positivo.

- Sin contraindicaciones para la implantación del BIB.

Se excluyó a pacientes con contraindicaciones para el uso del BIB $(14,15)$ :

- Hábitos tóxicos (alcoholismo o drogadicción).

- Embarazo o lactancia.

- Trastornos psiquiátricos.

- Alteraciones anatómicas, quirúrgicas o patológicas del tracto digestivo superior.

- Enfermedad digestiva o extradigestiva grave.

- Cirugía digestiva previa.

- Pacientes que precisaran tratamiento con aspirina, antiinflamatorios, anticoagulantes o esteroides.

Tabla I. Datos demográficos

\begin{tabular}{ll}
\hline Pacientes & 38 \\
\hline Hombres/mujeres & $11 / 27$ \\
\hline Edad media & $40,47(23-64)$ \\
\hline Peso inicial medio & $125,32(86,50-210)$ \\
& Mujeres 117,60 \\
& Hombres 144,25 \\
\hline IMC inicial medio & $47,25(34-80)$ \\
& Mujeres 47,17 \\
& Hombres 47,42 \\
\hline
\end{tabular}

Aquellos pacientes candidatos a la inserción del BIB ingresaron de manera protocolizada el día previo, realizándose analítica básica (hemograma completo, glucosa, urea, creatinina y coagulación) y preparación con dieta absoluta 8 horas previas a la implantación del BIB.

La implantación del BIB vía endoscópica se realizó en quirófano bajo anestesia general e intubación orotraqueal (16). El BIB fue llenado de forma estándar con $500 \mathrm{cc} \mathrm{de}$ suero salino mezclado con $10 \mathrm{ml}$ de azul de metileno para detectar precozmente una fuga mediante la coloración de la orina del paciente (17). Tras la colocación del BIB, se realizó un protocolo de tratamiento sintomático de posibles efectos secundarios precoces (náuseas, vómitos y 
dolor epigástrico generalmente), pautando tratamiento con antieméticos e inhibidores de la bomba de protones (IBP). Se instauró una dieta líquida con una progresión gradual a dieta sólida. Todos los pacientes permanecieron ingresados al menos 24 horas tras la implantación del BIB para observación y control de aparición de posibles efectos adversos. Posteriormente fueron dados de alta según evolución clínica y tolerancia oral a ingesta.

El seguimiento del paciente se realizó mensualmente por parte del Servicio de Endocrinología, con exploración física, analítica completa e incluyendo consejos higiénico-dietéticos: dieta hipocalórica de 1.000-1.500 calorías (compuesta por valores medios de $52,3 \%$ de carbohidratos, $19,5 \%$ de proteínas, $28,2 \%$ de lípidos, $18,12 \mathrm{~g}$ de fibra) (18), realizar ingestas frecuentes en pequeña cantidad (evitar comidas copiosas), evitar el decúbito prolongado tras ingesta y realizar ejercicio físico moderado (19).

La duración del tratamiento fue de 6 meses. La retirada del BIB se realizó bajo control endoscópico en quirófano, con anestesia general e intubación orotraqueal. Evaluamos la eficacia del tratamiento en términos de pérdida de peso inicial y disminución del IMC tras los 6 meses de tratamiento, así como la eficacia a medio plazo, determinando la pérdida de peso a los 6 y 12 meses postretirada del BIB.

Tras la retirada del BIB se realizó un cuestionario a cada paciente donde evaluamos:

1. Percepción del BIB: a) hiporexia; b) saciedad precoz; c) ambas; d) otras.

2. Molestias durante el tratamiento, medidas con una escala del 1 al 10 (siendo 1 mínimo y 10 máximo).

3. Efectividad subjetiva del tratamiento, también valorada con una escala del 1 al 10.

4. Recomendación del tratamiento a otros pacientes: a) sí; b) no.

\section{Análisis estadístico}

Estudio prospectivo observacional donde todos los resultados son expresados en medias (rangos) o porcentajes con un intervalo de confianza del 95\%. El análisis estadístico se realizó con el programa SPPS versión 15.0 para Windows.

\section{RESULTADOS}

La media de días de ingreso para la implantación del BIB fue de 2,82 días (2 días en el $36,8 \%$ de pacientes $[\mathrm{n}=$ $14], 3$ días en el 47,4\% de pacientes [ $n=18], 4$ días en el $13,2 \%$ de pacientes $[\mathrm{n}=5]$ y 5 días en el $2,6 \%$ de pacientes $[n=1])$. Los ingresos prolongados de 5 y 4 días se debieron a la aparición de efectos secundarios precoces tras la implantación del BIB (primera semana), tales como náuseas, vómitos y epigastralgia que retrasaron la tole- rancia oral a la ingesta y prolongaron la estancia hospitalaria.

En el $15,8 \%$ de pacientes $(n=6)$ se implantó el BIB como tratamiento puente a la cirugía bariátrica.

Las enfermedades asociadas con más frecuencia en nuestros pacientes fueron: hipertensión arterial (HTA) en un $23,7 \%$ pacientes $(n=9)$, diabetes mellitus $(D M)$ en un $21 \%$ de pacientes $(n=8)$, dislipemia en un $21 \%$ de pacientes $(n=8)$ y síndrome de apnea obstructiva del sueño (SAOS) en un $18,2 \%$ de pacientes $(n=7)$.

En el $89,5 \%$ de pacientes la duración del tratamiento con el BIB fue de 6 meses. En el resto de pacientes $(10,5 \%, \mathrm{n}=$ 4) no se pudo terminar el tratamiento en el tiempo previsto debido a la aparición de complicaciones: intolerancia digestiva severa sin respuesta a tratamiento farmacológico en 3 pacientes, requiriendo retirada precoz del BIB (en el primer mes, en el tercer mes y en el quinto mes de tratamiento respectivamente) y perforación gástrica con shock séptico secundario y fallecimiento en 1 paciente.

En los primeros días tras la implantación del BIB (primera semana) el $71,1 \%$ de los pacientes presentó náuseas, el 57,9\% vómitos y el 23,7\% de los pacientes epigastralgia. Estos efectos secundarios precoces disminuyen a lo largo del tratamiento, con buena respuesta al tratamiento farmacológico sintomático.

Existieron complicaciones en 7 pacientes $(18,4 \%)$, siendo en su mayoría complicaciones leves-moderadas (n =6), destacando 1 complicación grave (Tabla II). Las complicaciones observadas fueron: intolerancia digestiva severa en 4 pacientes, requiriendo 3 de estos pacientes retirada precoz del BIB; esofagitis moderada (grado II-III) en 2 pacientes, presentando uno de ellos una hemorragia digestiva alta leve y autolimitada tras la retirada del BIB; perforación gástrica en una paciente (en el primer mes de tratamiento), complicándose con shock séptico, falleciendo finalmente la paciente.

\section{Tabla II. Complicaciones del BIB}

\begin{tabular}{l}
\hline Complicaciones ( $\mathrm{n}=7$ pacientes, 18,4\%) \\
\hline Intolerancia digestiva $(\mathrm{n}=4$ pacientes) \\
Retirada precoz BIB ( $\mathrm{n}=3$ pacientes) \\
$\quad$ Primer mes \\
$\quad$ Tercer mes \\
$\quad$ Quinto mes \\
\hline Esofagitis moderada ( $\mathrm{n}=2$ pacientes) \\
Perforación gástrica $(\mathrm{n}=1$ paciente) \\
Shock séptico y muerte
\end{tabular}

Después de 6 meses de tratamiento con el BIB asociado a una dieta hipocalórica, la pérdida de peso media fue de $14,10 \mathrm{~kg}$ (0-46), siendo la pérdida de peso media en mujeres de $13,75 \mathrm{~kg}$ y en hombres de 14,93 kg (Fig. 1). La disminución media del IMC fue de $5,23 \mathrm{~kg} / \mathrm{m}^{2}(0-18)$, siendo la disminución media del IMC en mujeres de $5,49 \mathrm{~kg} / \mathrm{m}^{2}$ y en hombres de $4,63 \mathrm{~kg} / \mathrm{m}^{2}$ (Fig. 2). 


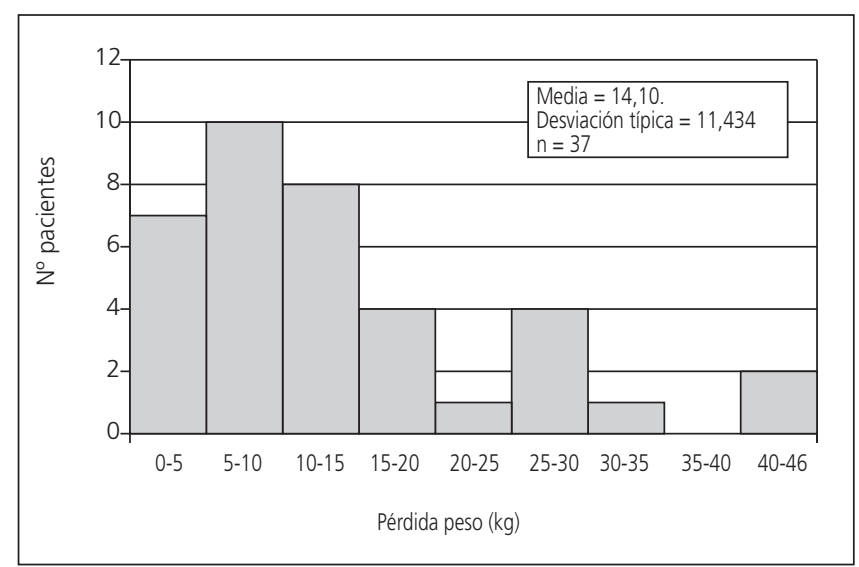

Fig. 1. Pérdida de peso en los pacientes tras 6 meses de tratamiento con el Bl.

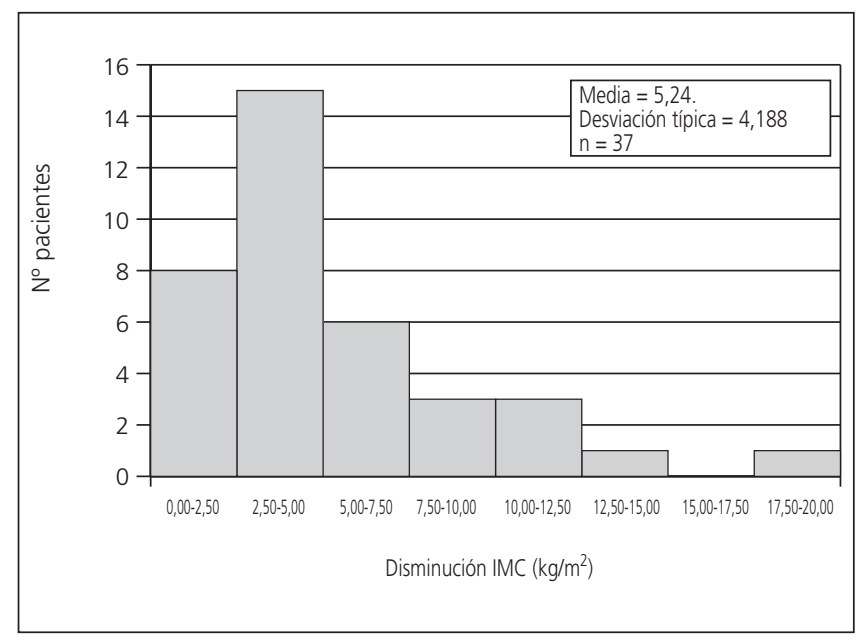

Fig. 2. Disminución del IMC en los pacientes tras 6 meses de tratamiento con el $\mathrm{BI}$.

En el cuestionario realizado por los pacientes tras el tratamiento evaluamos:

1. Percepción del BIB: el 47,4\% de los pacientes percibió saciedad precoz, el $36,8 \%$ de los pacientes otras sensaciones (ansiedad, hambre, náuseas y vómitos), el $5,3 \%$ hiporexia y el 7,9\% tanto hiporexia como saciedad precoz.

2. Molestias durante el tratamiento: la puntuación media en la escala semicuantitativa de las molestias percibidas fue de 4 , siendo en el 76,3\% de los pacientes valoradas con un número 5 o menor y en el $21,1 \%$ de pacientes valoradas con un número mayor de 5 .

3. Efectividad subjetiva del tratamiento: la media obtenida fue de 5,57. El 52,6\% de pacientes valoró la efectividad con un número mayor a 5 y el $44,7 \%$ con un número igual o menor a 5.

4. Recomendación del tratamiento: casi 2/3 de los pacientes (el $65,8 \%$ ) sí que recomendarían el tratamiento con el BIB a otros pacientes.
$\mathrm{Al}$ analizar la efectividad del tratamiento con el BIB a medio plazo (6 meses y 12 meses postretirada) se perdieron del seguimiento 7 pacientes: 1 paciente falleció tras perforación gástrica complicada con shock séptico, 6 pacientes por pérdida en el seguimiento endocrinológico, sin poder obtener la información clínica necesaria.

A los 6 meses postretirada del BIB, el 19,4\% de pacientes $(n=6)$ mantuvo la pérdida de peso, el $32,2 \%(n=$ 10) siguió perdiendo peso con una pérdida ponderal media de $5,4 \mathrm{~kg}$ y el $48,4 \%(\mathrm{n}=15)$ ganó peso con una ganancia ponderal media de $5,46 \mathrm{~kg}$.

A los 12 meses postretirada del BIB el 22,6\% de pacientes $(n=7)$ mantuvo la pérdida de peso, el $25,8 \%(n=$ 8 ) siguió perdiendo peso con una pérdida ponderal media de $9 \mathrm{~kg}$ y el $51,6 \%(\mathrm{n}=16)$ ganó peso con una ganancia ponderal media de $9,43 \mathrm{~kg}$.

\section{DISCUSIÓN}

Dentro del manejo multidisciplinar del tratamiento de la obesidad, la implantación del BI por vía endoscópica ha ganado popularidad en los últimos tiempos (20), debido a los resultados insatisfactorios del resto de opciones terapéuticas conservadoras (dieta hipocalórica, cambios de estilo de vida, fármacos, etc.). Las técnicas endoscópicas de implantación y retirada no son dificultosas, pero se recomienda un entrenamiento específico y la tolerancia del BI es globalmente aceptable (21).

La incidencia de efectos secundarios precoces (primera semana) en nuestra serie fue importante: un $71,1 \%$ presentó náuseas, un 57,9\% vómitos y el 23,7\% epigastralgia, siendo estos resultados similares a los reportados en otras series $(14,22,23)$. Estos síntomas mejoran en la mayoría de pacientes con tratamiento farmacológico y a lo largo del tratamiento. Sin embargo un $18,4 \%(\mathrm{n}=7)$ de pacientes sufrió complicaciones, siendo leves-moderadas en la mayoría de los casos $(n=6)$ : en 4 pacientes existió intolerancia digestiva, requiriendo en 3 pacientes retirada precoz del BIB; en 2 pacientes se objetivó esofagitis moderada tras la retirada del BIB. En otras series más amplias son recogidas otras complicaciones como oclusión intestinal, úlceras gástricas y desinflado del balón con migración espontánea $(8,17)$. Conviene destacar la existencia de 1 complicación grave: perforación gástrica, ya descrita en otros estudios publicados $(14,24)$, en la mayoría de los cuales existía una buena evolución clínica tras tratamiento quirúrgico. Sin embargo en nuestro estudio dicha perforación gástrica se complicó con shock séptico y muerte de la paciente. Dicha fatalidad ocurrió en el primer mes de tratamiento con el BIB, no existiendo en este caso complicaciones durante la técnica de implantación endoscópica del balón ni ingreso hospitalario prolongado (2 días de ingreso) por efectos secundarios precoces resistentes al tratamiento farmacológico. Se trataba de una paciente de alto riesgo con importantes enfermedades asociadas (HTA, DM y SAOS) y sin cumplimiento de la 
dieta hipocalórica ni los consejos higiénico-dietéticos. En el curso de la intervención quirúrgica urgente se objetivaron abundantísimos restos de alimentos en cavidad gástrica, sin poder determinar la causa de la perforación gástrica.

Se han comunicado otros casos de muerte durante el tratamiento de la obesidad con el BIB $(25,26)$. La mortalidad de nuestra serie no es desdeñable $(2,6 \%, \mathrm{n}=1$ paciente) pero debemos destacar que el tamaño muestral es pequeño. En otras series de tamaño muestral pequeño la mortalidad reseñada es similar (27), sin embargo en series amplias como la de los italianos Genco y cols. (2.515 pacientes) es menor (17).

Nuestros resultados refuerzan la necesidad de realizar correctamente una dieta hipocalórica y modificación de estilo de vida asociados al tratamiento con el BIB para conseguir una efectividad en términos de pérdida de peso, así como una mejor tolerancia y un menor número de complicaciones (28). Los criterios de selección de pacientes deben ser estrictos y debe realizarse un seguimiento adecuado del paciente durante el tratamiento.

En la mayoría de los pacientes existe un buen grado de aceptación al tratamiento con el BIB, encontrando en nuestra serie que un 76,3\% de los pacientes presentó molestias valoradas con un número igual o inferior a 5; y la sensación percibida en el $47,4 \%$ de pacientes fue saciedad precoz; resultados similares a los obtenidos en estudios previos (18).

La OMS recomienda una disminución de aproximadamente un $10 \%$ del peso corporal, ya que esta pérdida inicial de peso puede disminuir significativamente la severidad de los factores de riesgo asociados a la obesidad (29).

Los resultados obtenidos en nuestra serie muestran una buena evolución del IMC desde 47,25 hasta 42,06 kg/m². La media de pérdida de peso inicial tras 6 meses de tratamiento y la disminución media del IMC en nuestra serie fue similar a las obtenidas en las series de Loffredo y cols. y de Mathus-Vliegen y cols. $(10,18)$. La pérdida de peso individual fue extremadamente variable, comprendiendo un rango desde no pérdida de peso hasta pérdida de peso máxima de $46 \mathrm{~kg}$, resultando entonces una media de pérdida de peso de $14,10 \mathrm{~kg}$.

El éxito de esta técnica está relacionado directamente con la motivación del paciente y el correcto cumplimiento de la dieta hipocalórica asociada a modificaciones del estilo de vida $(28,30,31)$.

El mantenimiento de la pérdida de peso a medio y largo plazo es un tema controvertido y son pocos los estudios que lo evalúan. En nuestro estudio la mitad de los pacientes obesos $(51,6 \%)$ ganó peso 12 meses después de la retirada del BIB y el 48,4\% de los pacientes mantuvo la pérdida de peso o continuó perdiendo peso $(22,6$ y $25,8 \%$, respectivamente). Nuestros resultados en cuanto a mantenimiento de la pérdida de peso al año de retirado el balón son equiparables a los obtenidos en estudios previos $(10,32)$.
Por lo tanto el tratamiento de la obesidad con el BIB responde a los objetivos fijados por la OMS en cuanto a la pérdida inicial de peso (aproximadamente el 10\% del peso corporal) y a la eficacia, al menos parcial, del tratamiento a medio plazo (22).

En conclusión, el BIB asociado a una dieta hipocalórica y a un programa de modificación del comportamiento puede considerarse una opción terapéutica válida dentro del tratamiento multidisciplinar de la obesidad (33).

Es un tratamiento efectivo para obtener una pérdida de peso inicial motivante y un tratamiento seguro con una buena tolerancia global y un porcentaje bajo de complicaciones, aunque no exento de riesgos y complicaciones graves. Los beneficios tras la implantación del BIB parecen mantenerse a medio plazo (12 meses postretirada del BIB), aunque se hace necesario un seguimiento más prolongado para evaluar su efectividad a largo plazo.

La correcta selección de pacientes y el cumplimiento de la dieta con modificaciones en el estilo de vida por parte de estos son pilares básicos para obtener resultados óptimos.

\section{BIBLIOGRAFÍA}

1. Negrin S, Nguyen-Tang T, Dumonceau JM. Traitement endoscopique de l'obésité: le point sur le ballon intragastrique. Rev Med Suisse 2007; 3: 1958-61.

2. Clinical guidelines on the identification, evaluation and treatment of overweight and obesity in adults. The evidence report. National Heart, Lung and Blood Institute Education Initiative. Obes Res 1998; 6 (Supl. 2); 51S-209S.

3. Glenny AM, O'Meara S, Melville A, Sheldon TA, Wilson C. The treatment and prevention of obesity: A systematic review of the literature. Int J Obes Relat Metab Disord 1997; 21: 715-37.

4. De Waele B, Reynaert H, Urbain D, et al. Intragastric balloons for preoperative weight reduction. Obes Surg 2000; 10: 58-60.

5. Mathus-Vliegen EM, Tytgat GN. Intragastric balloon for treatmentresistant obesity: Safety, tolerance, and efficacy of 1-year balloon treatment followed by a 1-year balloon-free follow-up. Gastrointest Endosc 2005; 61: 19-27.

6. Doldi SB, Miceletto G, Perrini MN, et al. Intragastric balloon: Another option for treatment of obesity and morbid obesity. Hepatogastroentrology 2004; 51: 294-7.

7. Who needs an intragastric balloon for weight reduction? (editorial). Lancet 1988; II: 664 .

8. Alfalah H, Philippe B, Ghazal F, et al. Intragastric balloon for preoperative weight reduction in candidates for laparoscopic gastric bypass with massive obesity. Obes Surg 2006; 16: 147-50.

9. Nieben $\mathrm{OG}$, Harboe H. Intragastric balloon as an artificial bezoar for treatment of obesity. Lancet 1982; 1: 198-9.

10. Hogan RB, Johnston JH, Long BW, et al. A double-blind, randomized, sham-controlled trial of the gastric bubble for obesity. Gastrointest Endosc 1989; 35: 381-5.

11. Schapiro M, Benjamin S, Blackburn G, et al. Obesity and the gastric balloon: A comprehensive workshop. Gastrointest Endosc 1987; 33 : 323-7.

12. Doldi SB, Micheletto G, Perrini MN, et al. Treatment of morbid obesity with intragastric balloon in association with diet. Obes Surg 2002; 12: 583-7.

13. Galloro G, De Palma GD, Catanzano C, et al. Preliminary endoscopic technical report of a new silicone intragastric balloon in the treatment of morbid obesity. Obes Surg 1999; 9: 68-71.

14. Totté E, Hendrickx L, Pauwels M, et al. Weigth reduction by means of intragastric device: Experience with the Bioenterics Intragastric Balloon. Obes Surg 2001; 11: 519-23. 
15. Pérez-Cuadrado E, Silva C, Vázquez R, et al. Complicaciones de la prótesis balón intragástrica. Rev Esp Enferm Dig 1993; 84 (5): 2915 .

16. Wahlen $\mathrm{CH}$, Bastens B, Herve $\mathrm{J}$, et al. The Bioenterics intragastric balloon (BIB): How to use it. Obes Surg 2001; 11: 524-7.

17. Genco A, Bruni T, Doldi SB, et al. Bioenterics intragastric balloon: The Italian experience with 2,515 patients. Obes Surg 2005; 15: 1161-4.

18. Loffredo A, Cappuccio M, De Luca M, et al. Three years experience with a new intragastric balloon, and a preoperative test for success with restrictive surgery. Obes Surg 2001; 11: 330-3.

19. Zago S, Kornmuller AM, Agagliati D, et al. Benefit from Bio-enteric Intra-gastric balloon (BIB) to modify lifestyle and eating habits in severely obese patients eligible for bariatric surgery. Minerva Med 2006; 97: 51-64.

20. Galloro G, Sivero L, Magno L, et al. New technique for endoscopic removal of intragastric balloon placed for treatment of morbid obesity. Obes Surg 2007; 17: 658-62.

21. Doldi SB, Micheletto G, Di Prisco F, et al. Intrahastric balloon in obese patients. Obes Surg 2000; 10: 578-81.

22. Roman S, Napoleon B, Mion F, et al. Intragastric balloon for "nonmorbid" obesity: A retrospective evaluation of tolerance and efficacy. Obes Surg 2004; 14: 539-44.

23. Mion F, Gincul R, Roman S, et al. Tolerance and efficacy o fan airfilled balloon in non-morbidly obese patients: Results of a prospective multicenter study. Obes Surg 2007; 17: 764-9.
24. Laurent B, Charles D, Laurent M. Covered gastric perforation by the bioenterics intragastric balloon. J Clin Gastroentrol 2001; 33 (4).

25. Ballarè $\mathrm{M}$, Orsello $\mathrm{M}$, Del Piano $\mathrm{M}$. A case of death after insertion of an intragastric balloon for treatment of morbid obesity. Dig Liver Dis 2004; 36 (7): 499.

26. Timma N, Pomerantz I, Konikoff F. Intragastric balloon for morbid obesity. Harefuah 2006; 145 (11): 826-30, 861. Rewiev.

27. Spyropoulos C, Katsakoulis E, Mead N, et al. Intragastric balloon for high-risk super-obese patients: A prospective analysis of efficacy. Surg Obes Relat Dis 2007; 3 (1): 78-83.

28. De Waele B, Reynaert H, Van Nieuwenhove Y, et al. Endoscopic volume adjustment of intragastric balloons for intolerance. Obes Surg 2001; 11: 223-4.

29. World Health Organitation (WHO). Obesity: Preventing and managing the global epidemia. Report of a WHO consultation on obesity. Geneva: WHO; 1998.

30. Wright TA. Intragastric balloon in the treatment of patients with morbid obesity. Br J Surg 2002; 89: 489.

31. Mathus-Vliegen EMH. Treatment morbid and super-morbid obesity. Int J Gastroenterol 2005; 5: 9-12.

32. Herve J, Wahlen $\mathrm{CH}$, Schaeken A, et al. What becomes of patients one year after the intragastric balloon has been removed? Obes Surg 2005; 15: 864-70.

33. Al-Momen A, El-Mogy I. Intragastric balloon for obesity: A retrospective evaluation of tolerance and efficacy. Obes Surg 2005; 15 : 101-5. 